\title{
DAMPs and PDT-mediated photo-oxidative stress: exploring the unknown $\uparrow$
}

\author{
Abhishek D. Garg, ${ }^{a}$ Dmitri V. Krysko, ${ }^{b, c}$ Peter Vandenabeele ${ }^{b, c}$ and Patrizia Agostinis $* a$ \\ Received 2nd October 2010, Accepted 13th December 2010 \\ DOI: 10.1039/c0pp00294a
}

Damage-associated molecular patterns (DAMPs) or cell death associated molecular patterns (CDAMPs) are a subset of endogenous intracellular molecules that are normally hidden within living cells but become either passively released by primary and secondary necrotic cells or actively exposed and secreted by the dying cells. Once released, DAMPs are sensed by the innate immune system and act as activators of antigen-presenting cells (APCs) to stimulate innate and adaptive immunity. Cancer cells dying in response to a subset of conventional anticancer modalities exhibit a particular composition of DAMPs at their cell surface, which has been recently shown to be vital for the stimulation of the host immune system and the control of residual disease. Photodynamic therapy (PDT) for cancer has long been shown to be capable of killing malignant cells and concomitantly stimulate the host immune system, properties that are likely linked to its ability of inducing exposure/release of certain DAMPs. PDT, by evoking oxidative stress at specific subcellular sites through the light activation of organelle-associated photosensitizers, may be unique in incorporating tumour cells destruction and antitumor immune response in one therapeutic paradigm. Here we review the current knowledge about mechanisms and signalling cascades leading to the exposure of DAMPs at the cell surface or promoting their release, the cell death mechanism associated to these processes and its immunological consequences. We also discuss how certain PDT paradigms may yield therapies that optimally stimulate the immune system and lead to the discovery of new DAMPs.

\section{Introduction}

The immune system and the cells that help in its efficient working are a host's best defence against "foreign" pathogens/particles/ antigens which might prove to be detrimental (or even fatal) if not controlled in time. Usually our immune system can carry out this process with panache due to its ability to proficiently detect various molecular/biochemical patterns exhibited by a "foreign aggressor", e.g. 'Pathogen-associated Molecular Patterns (PAMPs)', expressed by various pathogens. ${ }^{1,2}$ These molecular patterns can be quite diverse in their biochemical nature, ranging from lipids and proteins to lipoproteins/glycolipids and glycoproteins. ${ }^{1,3}$ However, it has recently come to attention that, by using similar "molecular pattern detection senses", the immune system can detect the 'danger signals' associated with stressed or damaged cells in the body and deal with them accordingly. ${ }^{1-3}$ This process has been found in recent times to have implications in several medical disorders including autoimmune diseases and cancer. ${ }^{1,2}$

${ }^{a}$ Department of Molecular Cell Biology, Faculty of Medicine, Catholic University of Leuven, Campus Gasthuisberg ON1, Herestraat 49, B-3000, Leuven, Belgium. E-mail: patrizia.agostinis@med.kuleuven.be; Tel: +32.16 .345715$

${ }^{b}$ Molecular Signalling and Cell Death Unit, Department for Molecular Biomedical Research, VIB, Belgium

'Department of Biomedical Molecular Biology, Ghent University, Belgium $\dagger$ This article is published as part of a themed issue on immunological aspects and drug delivery technologies in PDT.
One of the earliest 'extensive' discussions in the direction of 'danger signals' was instigated by Polly Matzinger, when in the course of outlining her opinions over the 'self vs. nonself discrimination by immune system' she mentioned that cells exposed to chemical/physical/biological stress have the ability to emit 'danger signals', which are usually intracellular molecules (cytoplasmic, nuclear or membrane-associated) that are not secreted under normal conditions but, once released, could act as activators of antigen-presenting cells (APCs) like dendritic cells (DCs). ${ }^{4}$ Over the years however, the term 'danger signal' has been upgraded to the umbrella term 'Damage/Danger-associated Molecular Patterns (DAMPs) ${ }^{2,5}$ to outline the 'immuno-modulatory resemblance' of these molecules with the corresponding prokaryotic counterparts - PAMPs. Moreover, there have also been some additions to the definition of DAMPs/danger signals to accommodate the developments done over the years. As it stands currently, DAMPs are defined as mostly intracellular molecules (or molecules associated with cellular membranes in normal conditions) that are normally hidden within/with healthy cells, but tend to get exposed or secreted by damaged/dying cells such that once outside they have a propensity to acquire immunostimulatory/immunomodulatory properties. ${ }^{1,2}$ DAMPs that have been exposed or secreted have the ability, just like PAMPs, to interact with pattern-recognition receptors (PRRs) - both membrane-bound PRRs and intracellular PRRs. ${ }^{1,2}$ However, it is worth mentioning here that this definition of DAMPs cannot 
be considered to be 'exhaustive' since it is currently incapable of accommodating certain controversial or less studied signals like "whole organelle-based danger signals", e.g. complete mitochondria (capable of activating the NLRP3 inflammasome), ${ }^{6,7}$ or danger signals originating from "non-intracellular pools", e.g. those resulting from the extracellular matrix (ECM), and not to mention certain other types of danger signals/structures that have not been characterized completely yet, e.g. tumour cellderived exosomes (see Table 1). Thus, in future, if the existence of these DAMPs is proven to be incontrovertible then a new definition might come into calling; till that happens, in the present context (with respect to the well characterized immunomodulatory activities of other DAMPs) these danger signals might well be regarded as "atypical DAMPs". Alternatively, one could also define DAMPs in accordance with the cellular processes during which they are generated, e.g. Cell Death Associated Molecular patterns (CDAMPs), Apoptotic Cell Associated Molecular Patterns (ACAMPs) or cellular Stress Associated Molecular Patterns (SAMPs). These nomenclature/definition dilemmas bring us to an intriguing question, i.e. based on the type(s) of danger signals, can the immune system differentiate between the sources where they are coming from (i.e. injured tissue or stressed/dying/damaged cells)? Or does it always react to these danger signals in the same way (irrespective of the source)? The latter possibility is hinted towards (but not confirmed) by the fact that the receptors perceiving DAMPs are shared with those for PAMPs, ${ }^{1,2}$ however more research is required on the link between spectra of DAMPs and the cellular sources. Further research into this subject might be the only way to reach a proper nomenclature/classification scheme for DAMPs.

In recent times, several developments have been made in the direction of associating a particular DAMP or their spectra either with biochemistry of a specific cell death pathway or with particular chemotherapeutic stressors (or biological stress). These observations have not only influenced further exploration in the direction of immunogenic cell death but they have also led to a "race" in discovering/further characterizing new or already known therapeutic agents/modalities that are capable of sustaining a particular spectrum of DAMPs. ${ }^{2}$ One such therapeutic modality, which has known association with certain DAMPs but needs a fresh re-look on these lines is - photodynamic therapy (PDT) ${ }^{2,8-11}$ In the present review, we have discussed in detail how PDT has the potential to not only help us discover new DAMPs or better understand the exposure of known ones but also solve certain glitches/conundrums (discussed later) associated with them. Moreover, we have also reported certain observations on the link between PDT and certain emerging DAMPs obtained in our laboratory.

\section{DAMPs and the crossroads between cell death and immunogenicity}

DAMPs tend to mediate the immunogenicity of dying/damaged cells such that the type, diversity and mode of emergence (exposure, secretion or release) of DAMPs are intricately associated with the biochemistry of the particular cell death pathway in question. Most prominent DAMPs associated with various cell death pathways have been discussed in Table 1. In a 'classical' sense, the most immunogenic cell death pathway is considered to be necrosis, since rapid loss of plasma membrane integrity occurring during necrosis is always associated with the release of various pro-inflammatory factors. On the other hand, apoptosis in most cases is considered to be 'tolerogenic' and 'autophagic' cell death (i.e. cell death caused by autophagy) lacks a strongly definable immunogenicity. ${ }^{2,12-14}$ This sketch of cell death pathway immunogenicity has been modified by recent trends such as the further characterization of the pro-inflammatory profile of necrosis and its diversity (Table 1), the emergence of 'immunogenic apoptosis' as a new 'subset' of apoptosis and autophagic cell death has shown the ability to release/expose DAMPs (discussed in detail elsewhere). ${ }^{2,12-14}$

In therapeutic terms, the immunogenicity of apoptosis is preferable for application rather than necrosis (or for that matter autophagic cell death) since necrosis can lead to harmful immunological reactions ${ }^{15}$ (on the other hand, the extent of immunological impact of autophagic cell death is as yet uncharacterized, thereby making it an uncertain modality to use in the context of 'immunochemotherapy')., ${ }^{2,8}$ Immunogenic apoptosis is a phenomenon that has emerged recently and tends to be moreor-less stressor dependent (as demonstrated for anthracyclines, oxaliplatin, UVC light, $\gamma$-irradiation and bortezomib).,12,14,16 One biochemical feature that has been found to be common amongst all these agents is the ability to induce Endoplasmic Reticulum (ER) directed stress either as a primary or secondary effect., ${ }^{2,12,14,16}$ Here, ER stress is a condition where the normal ER homeostasis is disturbed by chemical/biological/chemotherapeutic stressors (e.g. viral infections, oxidative injury, hypoglycaemia, hypoxia, thapsigargin) thereby leading to imbalance between protein folding capacity and load. ${ }^{17}$ Moreover, in the case of agents like anthracyclines, the involvement of ER stress probably associated with the production of reactive oxygen species or ROS has been implicated. ${ }^{14,16}$ Considering that ER stress has already been known to associate with immunological signalling, these new observations linking it with 'critical' DAMPs and immunogenic apoptosis are intriguing. For more on this link between ER stress-based cell death, inflammation and DAMPs, please refer to the recent reviews by Verfaillie et al. ${ }^{17}$ Hummasti \& Hotamisligil, ${ }^{18}$ Hotamisligil ${ }^{19}$ and Kaser \& Blumberg. ${ }^{20}$

Biochemically speaking, immunogenic apoptosis is supposed to have all the main hallmarks of 'physiological' apoptosis however the main difference is in the immunological profile which is immunostimulatory rather than tolerogenic. On these lines, immunogenic apoptosis can be considered to possess two main properties beyond 'physiological' apoptosis, i.e. (1) exposure/secretion of (critical) 'immunogenic signals' or DAMPs and (2) ability to activate the immune system (so as to cause maturation of antigen-presenting cells which then might prime the adaptive immune cells against the target cell antigens)., $2,8,12,14,16,21$ However exposure/secretion of 'critical' DAMPs is one of the most vital properties for immunogenic apoptosis as proved in the case of apoptotic DAMPs like surface calreticulin (ectoCRT), surface HSP90 (ecto-HSP90) and extracellular ATP (exoATP) (Table 1). The emergence of concepts associated with DAMPs have opened up new vistas which might have effects on both sides of the line in terms of disease progression, a subject that has been dealt with briefly in the next section. 
Table 1 Major Damage-associated Molecular Patterns (DAMPs) linked with necrosis, apoptosis and cell death accompanied by/caused by autophagy and their association with PDT

\begin{tabular}{|c|c|}
\hline Molecule/Component & Immunomodulatory activity \\
\hline \multicolumn{2}{|c|}{ Surface (outer leaflet of plasma membrane) exposed DAMPs } \\
\hline $\begin{array}{l}\text { Phosphatidylserine } \\
\text { (PS) }\end{array}$ & $\begin{array}{l}\text { PS normally resides in the inner leaflet of the plasma membrane, however } \\
\text { damaged/dying cells "scramble" it to the outer leaflet where it acts as an } \\
\text { important "eat me' signal and interacts with multiple immune cells } \\
\text { receptors to help in efficient phagocytosis and induction of } \\
\text { anti-inflammatory response. Also interacts with opsonins like: Annexin-V, } \\
\beta 2 \text {-glycoprotein ( } \beta 2 \text { GP1), milk fat globule EGF/factor VIIC (MFG-E8) } \\
\text { and growth arrest-specific gene } 6 \text { (Gas6). }\end{array}$ \\
\hline $\begin{array}{l}\text { Heat Shock Proteins } \\
\text { (HSPs) }\end{array}$ & $\begin{array}{l}\text { HSPs normally reside in various intracellular regions/organelles, however } \\
\text { under certain stresses, they have been known to be exposed on the } \\
\text { damaged/dying cell's surface and mediate immunomodulatory processes. } \\
\text { More specifically, surface-exposed HSP70 and HSP90 have been found to } \\
\text { affect phagocytosis and antigen processing/presentation. HSP90 has been } \\
\text { found to define immunogenicity of dying cells. }\end{array}$ \\
\hline
\end{tabular}

Calreticulin (CRT) CRT normally resides in diverse intracellular regions/organelles, however under certain particular stresses (mostly ER-directed) its presence on the plasma membrane tends to enhance "strongly" (sometimes even before PS exposure). Once on the plasma membrane, ecto-CRT has been shown to act as an 'eat me' signal as well as assist in 'increasing' the immunogenicity of the dying cells.

Oxidized

Phosphatidylcholine

Ox-PtC has been reported to emerge specifically on the surface of the (Ox-PtC) apoptotic cells and postulated to play a role in their phagocytic clearance (possibly via CD36).

\section{Extracellularly released/secreted DAMPs}

Heat Shock Proteins (HSPs)

HSPs have been known (more often) to be released passively from dying cells, however there is an instance reported of active release via a non-classical secretory pathway as well. Secreted HSPs can act as carriers of crucial tumour antigens, helping in their proper uptake and processing by APCs. They can also trigger secretion of various pro-inflammatory cytokines from immune cells. Recently it was shown that extracellular HSP90 $\beta$ can inhibit activation of latent TGF- $\beta 1$.

Peroxiredoxin $1(\operatorname{Prx} 1)$

Prx 1 can be passively released by dying cells, however there is evidence towards active secretion via non-classical secretory pathway in non-small cell lung cancer cells. It was recently shown that "secreted" Prx1 can stimulate TLR4-dependent cytokine secretion (in DCs and macrophages).

Calreticulin (CRT)

Extracellular CRT (exo-CRT) tends to act as an important chemotactic agent as well as assists in wound healing.

HMGB1

HMGB1 normally resides within the nucleus however it has been reported that necrotic and secondary necrotic cells might release HMGB1 passively, while cells dying with autophagy under the stress of recombinant fusion protein diphtheria toxin-epidermal growth factor (DT-EGF) have been reported to secrete HMGB1 actively. HMGB1 has a very prominent cytokine-like property and tends to stimulate immune cells into producing various pro-inflammatory cytokines.

BCL2

BCL2 mediates various intracellular functions, especially those relating to regulation of cell death. It was recently reported that extracellular BCL2 might act as a cytoprotective DAMP, thereby reducing apoptosis and tissue injury.

ATP, UTP

ATP/UTP are normally confined pre-dominantly in the intracellular space, however it has been reported that they might be released passively by necrotic cells and actively by apoptotic cells dying under particular stresses. Extracellular ATP and UTP have the ability to assist in chemoattraction of immune cells. In fact, ATP has been shown to activate the NLRP3 inflammasome in macrophages/dendritic cells.

Annexin A1

Normally confined to internal space, annexin A1 has been observed to be externalized on secondary necrotic cells (but not primary apoptotic) and cause prevention of cytokine production in macrophages. Annexin A1 is known to be potently anti-inflammatory in nature.

HSPs like HSP70, HSP60, GRP94 and GRP78 have

been associated with

Photofrin-PDT (Fig. 1).

Hypericin-PDT can induce ecto-CRT exposure (Garg et al., unpublished data)

(Fig. 2).

Not known

Passive release of HSP70 has been associated with Photofrin-PDT (Fig. 1).

Not known

Not known

Not known

Not known

Hypericin-PDT can induce exo-ATP secretion (Garg et al., unpublished data).

Not known 72 
Table 1 (Contd.)

\begin{tabular}{ll}
\hline Molecule/Component & Immunomodulatory activity \\
\hline $\begin{array}{l}\text { Other major } \\
\text { extracellular DAMPs }\end{array}$ & $\begin{array}{l}\text { Endothelial Monocyte-activating Polypeptide II (EMAPII), Cross-linked } \\
\text { dimer of ribosomal protein S19 (dRP S19), lysophosphatidylcholine } \\
\text { associated with }\end{array}$ \\
$\begin{array}{l}\text { (LPC) and fragments of human tyrosyl tR NA synthetase (TyrRS) are } \\
\text { some of the 'find me' signals released from apoptotic cells (either actively } \\
\text { or passively depending on the conditions). Acting as a chemotactic factor } \\
\text { for attracting various immune cells is a major extracellular function of } \\
\text { most of them. LPC can also assist in DC maturation. }\end{array}$
\end{tabular}

Other major extracellular DAMPs associated with necrosis
S100/Calgranulin protein family members (S100A8, S100A9, S100A12), Hepatoma-derived Growth Factor (HDGF), monosodium urate (MSU), SAP130, galectins, thioredoxin and cathelicidins are 'find me' signals passively released by necrotic cells. These molecules mediate a number of processes ranging from attracting various immune cells (chemotactic factor) and interacting with vital immune cell receptors (like TLR4/RAGE) to inducing secretion of pro-inflammatory cytokines/enzymes from immune cells.

\section{End-stage products based DAMPs}

Genomic DNA, mRNA, snRNPs

They are usually released by dead cells (necrotic or secondary necrotic) and tend to activate various pro-inflammatory processes.

Mitochondrial DAMPs (mtDAMPs)

DAMPs derived from mitochondria (like mitochondrial DNA and $\mathrm{N}$-formyl peptides) have been shown to be capable of activating strong inflammatory response, however their relevance in a cell death modality set-up is yet to be fully tested. Recently it was reported that even whole mitochondria released from necrotic cells are capable of activating the inflammasome.

\section{"Atypical DAMPs" originating from Extracellular Matrix (ECM) components}

Biglycan Interacts with TLR2/4 and $\mathrm{P} 2 \mathrm{X}_{4} / \mathrm{P} 2 \mathrm{X}_{7}$ receptors on macrophages and induces NLRP3/ASC inflammasome-dependent IL-1 $\beta$ production. It can also interact with various cytokines.

Hyaluronan Similar to biglycan, hyaluronan has been reported to interact with TLR2/4 receptors as well as activate NLRP3 inflammasome-dependent IL-1 $\beta$ production.

Versican

Versican was recently reported to interact with the TLR2/6/CD14 complex thereby activating tumour-infiltrating myeloid cells and assisting in tumour metastasis.

Fibrinogen

A known ligand for TLR4 that has been shown to activate macrophage's chemokine synthesis program.

Heparan Sulfate fragments

Acts as a TLR4 ligand on DCs and assists in their maturation as well as later T-cell stimulation.

Fibronectin extra domain A

Known to activate expression of genes associated with TLR4-dependent inflammatory response.

Promotes TLR4-dependent synthesis of inflammatory cytokines.

Tenascin-C

"Uncharacterized" DAMPs

Tumour cell-derived exosomes
Exosomes are small lipid-bilayer vesicles reported to be released from tumour cells recently. Exosomes are considered to be strongly immuno-modulatory since they are enriched in heat shock proteins (like HSP70 and HSP90), tetraspanins and MHC complexes. It has been reported that DCs can use exosomes for proper cross-presentation and subsequent $\mathrm{T}$ cell activation. In fact, it has been a common knowledge for some time that DCs secrete exosomes and use them as "communication vehicles" amongst themselves. However the exact "DAMP-ness" of exosomes (with respect to dying/stressed cells) is yet to be characterized fully.
Association with PDT

Ref.

Not known

2,13

Not known

Not known

Not known

$7,73,74$

Not known

Not known

76

Not known

Not known

Not known

Not known

Not known

Not known

78,79

Note: Here "passive" refers to release in presence of plasma membrane permeabilization while "active" means secretion/surface exposure in presence of negligible/non-significant plasma membrane permeabilization. 


\section{DAMPs: from bench-side research to bed-side reality}

DAMPs are exposed, secreted actively or released passively in a manner that depends on the type of damage or type of stressors, as discussed previously. Once in the extracellular space, DAMPs mediate various immunological processes. However the point to ponder is whether the immunological processes activated by DAMPs help 'against' a disease's progression or favour its progression (since DAMPs can be immunogenic/proinflammatory as well as anti-inflammatory)? Processes associated with certain DAMPs (e.g. in the case of exo-ATP and ectoCRT) have been recently shown to be capable of uniting tumour cell kill and revival of anti-tumour immunity within a 'single paradigm' thereby opening up a new area in our 'therapeutic war' against cancer. ${ }^{2}$ On the other hand however, certain DAMPs (e.g. HMGB1) have been suspected to promote the tumour-driven inflammatory environment as well as the processes driving inflammatory or autoimmune diseases ${ }^{15}$ (discussed later briefly). Either way, the knowledge we gain about DAMPs and their mechanism/kinetics could help us in bettering our treatment strategies against certain diseased conditions in clinical settings.

Up front, knowledge about DAMPs could help us in making proper decisions about which chemotherapeutic drugs should be more or less preferred for a particular condition. Also, presence (or absence) of particular DAMPs could serve the purpose of being 'biomarkers' thereby assisting in diagnostics or prognostics either towards identification of the disease's stage or towards the identification of the extent of inflammation associated with that disease. For example, it was recently shown in stage IIIB colon cancer patients that higher expression levels of the DAMP calreticulin were associated with increased tumour-infiltration by T-lymphocytes, both of which in turn where postulated to be associated with higher 5 year survival rate of patients. ${ }^{22}$ Apart from this, knowledge about DAMPs can also help us in improving certain vaccination strategies employed against cancer. For instance, 'autologous' (killed) tumour cell based-vaccines ${ }^{23,24}$ and 'autologous' dendritic cell (DC)-based vaccines ${ }^{25,26}$ have shown considerable promise mitigated by instances of failures within clinical settings. ${ }^{27,28}$ Currently, such vaccine preparations mostly involve simple lysis or heat shock-based killing of tumour cells, treatments which might not be 'strongly' immunogenic. However, our knowledge of treatment strategies that induce immunogenic apoptosis associated with crucial DAMPs could help us in applying them in preparation of the above-mentioned vaccines so as to improve their adaptive immune system priming abilities. Also, one may go one step further and add a purified version of particular DAMPs to the vaccine preparations in order to improve the 'immunogenicity' of the vaccine.

As evident from the above discussion, in terms of DAMPs, there are several things that are of utmost importance for further investigation to completely exploit their clinical potential, such as: further search for agents/modalities capable of inducing immunogenic cell death, characterization of new (hopefully more crucial) DAMPs and identification of molecular pathways behind active surface exposure/secretion of DAMPs. In the following sections, the DAMPs associated with PDT and the possibilities offered by PDT in bettering the DAMP-based paradigms have been discussed in detail.

\section{DAMPs and PDT: a revival in the calling!}

The link between PDT and DAMPs is in no way new, as there are already 'solid' as well as promising observations available about not only association of particular DAMPs with PDT but also the immunogenicity of PDT-treated cancer cells, as well as the ability of PDT-treated cancer cells to help in 'revival' of antitumour immunity both in pre-clinical as well as clinical settings. ${ }^{8}$ For more on PDT and the immunological processes activated by it, please refer to reviews by Gollnick \& Brackett, ${ }^{29} \mathrm{Garg}$ et al. ${ }^{8}$ and Castano et al. ${ }^{30} \mathrm{It}$ is well-known that PDT-killed tumour cells tend to induce stronger anti-tumour immunity in vivo than tumour cell lysates produced via treatments like ionizing irradiation, freezethaw or UV irradiation. ${ }^{8}$ Based on these premises PDT-based whole tumour cell killed vaccines have been produced and have shown good promise in pre-clinical models (and led to phase I clinical trial on similar lines), ${ }^{11,29}$ thereby further substantiating the stand that PDT-treated cancer cells are highly immunogenic. Such observations were the primary reasons in the past which lead researchers to probe the link between PDT and DAMPs.

It is well known that PDT is capable of inducing all three types of cell death modalities, i.e. necrosis, 'autophagic' cell death and apoptosis, ${ }^{31}$ however what would be interesting to confirm is whether the concept of 'PDT-induced cell death associated DAMPs' exists. ${ }^{8}$ For necrosis, (inducing) agent-to-agent variations have not been reported to change the spectra of necrotic DAMPs. Thus, although PDT-induced necrosis would not be expected to have significantly different spectra of DAMPs, it might well be associated with DAMPs that are more chemically modified under oxidative stress. However, this latter possibility has not been investigated yet. It is known now that the immune cells in terms of phagocytosis deal with necrosis in a different manner than apoptosis,,$^{32}$ however whether this also applies to antigen processing is a matter of debate. Thus in clinical settings, due to very limited amount of data, it is tough to speculate or propose if PDT-induced necrosis would be harmful or beneficial. If the DAMP spectra associated with PDT-induced necrosis is found to be "biochemically different" from normal necrosis then there is a possibility of it being beneficial but this needs further investigation. Similarly, nothing substantial is known about the spectra of DAMPs associated with PDT-induced autophagic cell death. On the other hand, PDT-treated apoptotic cells have been predominantly associated with heat shock protein (HSP)based DAMPs. HSPs are considered to be a well-known group of DAMPs ${ }^{33}$ due to their high hydrophobicity, a biochemical character considered to be critical for DAMP-like behaviour. ${ }^{3}$ HSPs are considered to be 'strongly' immunostimulatory in all extracellular forms, i.e. surface-exposed, secreted (actively or passively) and in the form of passively-released complexes with lipid membranes/tumour-derived peptides (Table 1). For more on DAMP-like activity and immunomodulatory potential of HSPs, please see reviews by Pockley, ${ }^{34}$ Tsan \& Gao ${ }^{33}$ and Garg et al. ${ }^{2,8}$ In the context of PDT, it has been shown that Photofrin-PDTtreated SCCVII cancer cells can surface expose molecules like ectoHSP70, ecto-HSP60 and ecto-GRP94 (GRP - glucose-regulated protein) (Fig. 1) more robustly in apoptotic state rather than in healthy conditions. ${ }^{35}$ Moreover, these PDT-treated cells were also found to secrete exo-HSP70, a DAMP that according to present knowledge is suspected to be released passively, i.e. after 


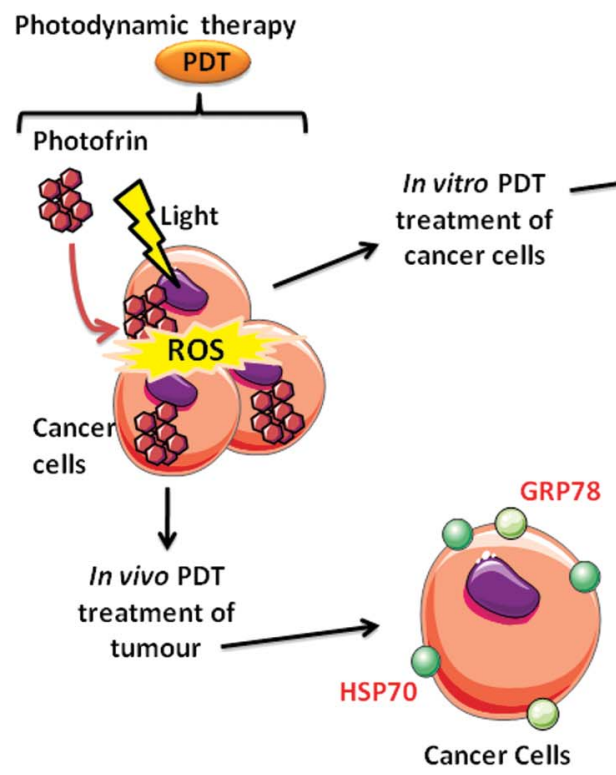

PDT-treated Tumour-derived cells:

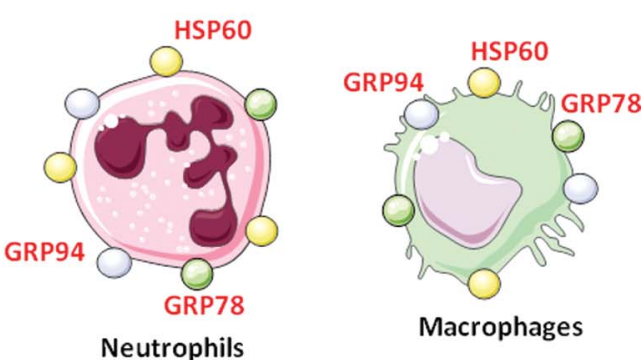

Fig. 1 DAMPs associated with PDT. It has been shown that Photofrin-PDT leads to different DAMP spectra on the surface of same cancer cells depending on the treatment setting, i.e. in vitro or in vivo. In in vitro setting, Photofrin-PDT causes surface exposure of HSP60, HSP70 and GRP94 on the treated cancer cells accompanied/followed by extracellular release of HSP70. However, in in vivo settings, Photofrin-PDT causes HSP70 and GRP78 surface exposure on the treated cancer cells. On the other hand, neutrophils and macrophages derived from the same Photofrin-PDT treated tumour site tend to expose HSP60, GRP94 and GRP78 on their surface.

significant membrane permeabilization. ${ }^{35}$ Intriguingly, when the authors extended these in vitro experiments to in vivo settings, they found that the spectrum of DAMPs exposed on the PDTtreated SCCVII tumour cells was different such that while they still engaged ecto-HSP70 they no longer exposed ecto-HSP60 and ecto-GRP94, but exposed yet another molecule, i.e. GRP78, on their surface (Fig. 1). ${ }^{35}$ To our knowledge, this is the only study where surface-exposed DAMPs have been reported to differ in the same cancer cells between in vitro and in vivo treated settings. Whether these effects are specific for PDT or can be extended to other modalities is an open question. Apart from that, these authors also reported that neutrophils and macrophages derived from the PDT-treated tumour microenvironment surface-exposed molecules like HSP60, GRP78 and GRP94 (Fig. 1), ${ }^{35}$ thereby further implying the possibility of variations in emergence of DAMPs based on cell-type differences. These authors implicated ecto-HSP70 in supporting the opsonisation of cancer cells by the C3 complement protein. ${ }^{11}$ It was later reported that PhotofrinPDT-based ecto-HSP70 exposure tends to relate closely with the changes in mitochondrial transmembrane potential. ${ }^{36}$ Depending upon the type of cell death induced by PDT, the associated DAMP spectra can lead to various effects ranging from strong proinflammatory reaction (which might be harmful in certain cases) to robust pro-inflammatory reaction which can help in priming of the immune system against the tumour antigens. ${ }^{12,31,37}$ Clinically, the latter is preferred; however, since the knowledge about PDTassociated DAMP spectra is limited, it is tough to envisage the clinical PDT settings that might be applied to produce a certain beneficial effect. This deserves further attention in near future.

The PDT-DAMP link discussed above has not been re-analyzed in the light of latest evidence that has emerged in the field of DAMPs, both in terms of the emerging DAMPs as well as the molecular pathways involved in active exposure/secretion of emerging and well-known DAMPs. In the following sections, we discuss how PDT might lead the way ahead in terms of DAMPs and immunogenic cell death.

\section{DAMPs and photo-oxidative stress: how can PDT lead the way ahead?}

It would be fitting to postulate that what we know currently about DAMPs, as a whole, is only a tip of the iceberg. Thus, needless to say, several conundrums and glitches associated with DAMPs have either not received enough attention or have not been fully answered (e.g. how does oxidative modifications affect DAMPs? What are the 'pools-of-origin' of various DAMPs? What molecular components are involved in active exposure/secretion of DAMPs? What is the role of autophagy in DAMPs exposure/secretion?). We envisage that the unique nature of PDT might help us solve some of them if not all (or at least provide valuable inputs). In the following sub-sections we have dealt with certain scenarios in which PDT can 'come to the rescue' (or has already been shown to be of major importance).

\section{Organelle-directed stress and DAMP translocation/secretion mechanisms: how can PDT enlighten us?}

Considering the available knowledge underlying the release mechanisms, there exist more (characterized) DAMPs which are passively released by dying/damaged cells than actively exposed/secreted (see Table 1). However this difference might not be because of DAMP molecules' preference towards a particular extracellular domain but rather due to our limited knowledge of DAMPs as well as differences in biochemistry of a particular 

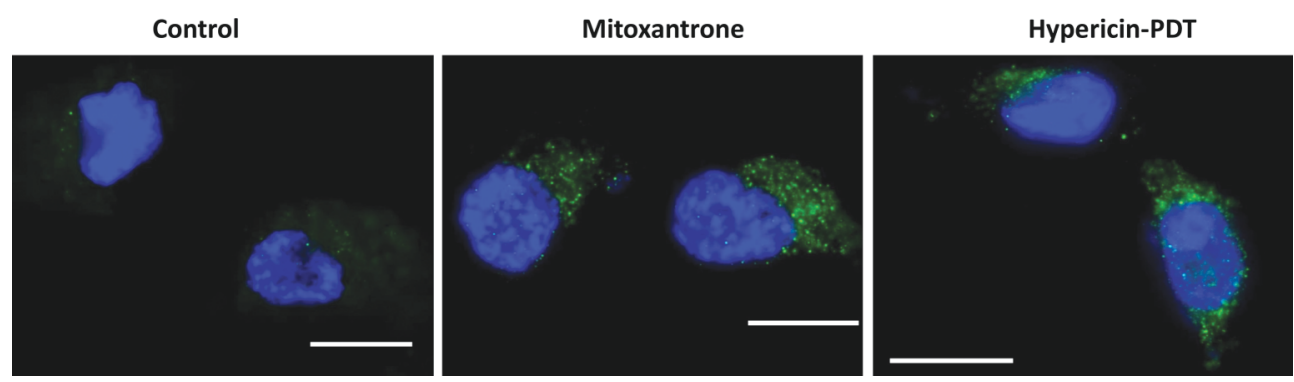

Fig. 2 Photo-oxidative ER stress via hypericin-PDT causes surface exposure of calreticulin (ecto-CRT). T24 human bladder carcinoma cells were treated with Mitoxantrone/MTX ( $1 \mu \mathrm{M}$ MTX for $4 \mathrm{~h}$ ) and Hypericin-PDT ( $150 \mathrm{nM}$ hypericin for $16 \mathrm{~h}$ and $2.16 \mathrm{~J} \mathrm{~cm}^{-2}$ irradiation with visible light followed by recovery at $1 \mathrm{~h}$ post-PDT). MTX is the positive control for ecto-CRT exposure. After treatment, the cells were fixed and immunostained for ecto-CRT (primary antibody: rabbit anti-calreticulin polyclonal antibody, cell signaling; secondary antibody: Alexa Fluor-488 goat anti-rabbit IgG, Invitrogen) followed by DAPI-based staining for the nucleus. Scale bar $=15 \mu \mathrm{m}$.

cell death pathway. Nevertheless, while molecular pathways for some actively translocated/secreted DAMPs have been described yet for other they are either incomplete or less characterized. Ecto-CRT remains one of the DAMPs for which a molecular pathway has been described in some detail, ${ }^{14}$ but there are still areas within that pathway that need further attention. One thing that emerged from the studies on ecto-CRT is that the 'pool-oforigin' of DAMPs from within a sub-cellular location of a cell might finally influence the type of pathway that the corresponding DAMP eventually follows. For example, ER stress in the case of ecto-CRT sets the tone for molecular components of the secretory pathway and the Unfolded Protein Response (UPR) signalling to play a role in translocating CRT to the surface. ${ }^{14,16,21}$ However, in this case, the agents used for exerting ER stress-mediated ecto-CRT translocation (especially anthracyclines) are known to produce ROS as a secondary effect of cellular damage such that their 'action' is not exclusively directed towards ER but also other organelles. ${ }^{38}$ Thus, to further characterize this pathway we need a stressor that has ROS-based ER stress as its primary effect. Similarly, in the case of other DAMPs like exo-ATP, ecto-/exoHSP70, ecto-HSP90, not only the 'pool-of-origin' is elusive but also the exact molecular components involved are unknown.

The uniqueness of PDT as a therapy could be of vital importance here, both for further characterization of molecular pathways behind DAMP translocation/secretion and for the identification of their 'pool-of-origin'. PDT and its effects heavily depend upon the sub-cellular localization of the photosensitiser (PS) and luckily there is a significant panel of photosensitisers available with various different sub-cellular localization profiles. ${ }^{31}$ Moreover, ROS-based stress directed towards the organelle where the photosensitiser localizes is a primary effect of PDT, e.g. hypericin (localizes predominantly in the ER)-based PDT would produce photo-oxidative ER (p-ox ER stress) stress while 5ALA (localizes in the mitochondria)-based PDT would produce photo-oxidative mitochondrial stress. ${ }^{30,31}$ It is worth mentioning here that correlation between a particular spectrum of DAMPs and stress on a particular sub-cellular site or organelle is a relatively new and highly unexplored concept. For instance, based on the observations showing that ER-directed and ROS-based stress causes surface exposure of ecto-CRT, it is presumable that ecto-HSP70 exposed after Photofrin-PDT requires pathways originating from the subcellular sites where Photofrin-PDT exerts its primary photodamage, ${ }^{39,40}$ namely the ER, mitochondria or
Golgi complex. However, further investigations are required to establish such correlations, since these can influence further studies on molecular pathways.

To this end, we have been investigating the DAMP spectra associated with photo-oxidative ER stress (p-ox ER stress) exerted by especially hypericin-based PDT (Hyp-PDT) in our laboratory. ${ }^{41}$ We have observed that p-ox ER stress induces 'pre-apoptotic' enhancement in surface exposure of calreticulin (ecto-CRT) (see Fig. 2) as quickly as $30 \mathrm{~min}$ after PDT in a dose-dependent manner, in T24 human bladder carcinoma cells (Garg et al., unpublished results). Interestingly, Photofrin-PDT, although shown to be capable of exerting ROS-based ER stress ${ }^{40}$ does not incite enhanced surface exposure of ecto-CRT in the same cells (J. Golab, personal communication). This difference between photofrin-PDT and hypericin-PDT is rather intriguing and might be explained by the magnitude of p-ox ER stress generated since photofrin localizes in the ER to a lesser extent than hypericin, or by distinct PDTmediated effects on processes required to translocate CRT from the ER to the plasma membrane. We also observed that Hyp-PDT induces 'pre-apoptotic' active exo-ATP secretion and late stage passive release of DAMPs like HSP70, HSP90 and CRT (Garg et al., unpublished results). Overall these observations reveal the potential of Hyp-PDT in causing exposure/secretion of 'critical' DAMPs and also adds this modality in a rather 'small club' of anti-cancerous therapeutic agents/modalities capable of exposing immunogenic signals like ecto-CRT. ${ }^{14,16}$

\section{Oxidative modifications of DAMPs: to be or not to be?}

The possibility of immunomodulatory/immunostimulatory potential of a DAMP being neutralized via oxidative modifications is a debate that has risen recently. Apparently, under natural conditions, the sub-cellular location from where a DAMP is derived can finally decide the kind of modification it might undergo once outside the cell. ${ }^{37,42}$ The redox variations within the cells and outside mostly are defined by the thiol redox status such that the cytosol is highly reducing (due to thiol-regulating enzymes like thioredoxin-thioredoxin reductase; reduced and oxidized glutathione molecules maintained in a reducing 100:1 ratio), lumens of all secretory pathway components including ER is highly oxidizing (reduced and oxidized glutathione molecules maintained in an oxidizing $3: 1$ ratio), nucleus is highly reducing while the extracellular space is strongly oxidizing (due to 
the number of reducing enzymes and abundance of oxidizing agents) ${ }^{37,42}$ Understandably, the proteins from these sub-cellular regions are accustomed to the redox status of that region such that proteins in the cytosol and nucleus are highly susceptible to oxidation-based neutralization due to presence of cysteine residues with free sulfhydryl groups. On the other hand, proteins derived from secretory pathway components like the ER are not because their cysteine residues are linked by disulfide bonds, thereby providing extra stability in oxidizing environments. ${ }^{42}$ Thus DAMPs derived from nucleus and cytosol might be more susceptible to extracellular space-based oxidation than those derived from the Golgi or the ER, a claim substantiated by certain recent observations. For instance, a predominantly cytosolic protein, S100A8 (Table 1), which tends to act as a DAMP when secreted and stimulates recruitment of myeloid cells to the site of inflammation, can be oxidatively neutralized such that the oxidized form is chemotactically inactive. ${ }^{43}$ Similarly, HMGB1, a well-known DAMP originating from the nucleus (Table 1), has been shown to be susceptible to oxidation-based neutralization ${ }^{44}$ although not in the context of extracellular spacebased oxidation but rather oxidation via ROS produced from mitochondria because of caspase activity. The latter work also reasoned that the type of cell death pathway may also govern the oxidative state of certain DAMPs, e.g. caspase activation during apoptosis causes mitochondria-instigated HMGB1 oxidation ${ }^{44}$ but probably not under necrosis, although the mechanism of this differential outcome between apoptosis and necrosis remains enigmatic. However, in the case of cells dying through necrosis, the extracellular oxidative environment comes into the picture, thereby making this a moot case.

Therefore the debate centers around some still unresolved questions like: does oxidation-based neutralization theory apply to all DAMPs? This discussion becomes intriguing if we drag a ROS-based modality like PDT into the picture since ROS produced by PDT might be expected to affect DAMPs. While this might be considered puzzling, evidence derived from PDT treatments both in vitro and in vivo points towards anything but inactivation of immunostimulation. ${ }^{8}$ PDT-treated cancer cells have been reported to be 'strongly' immunogenic and PDTderived HSPs are usually observed to remain immunostimulatory. ${ }^{8}$ In fact, it has been proposed that photo-oxidation via PDT might actually be immunologically beneficial such that PDT-based protein unfolding events and membrane changes might reveal certain neo or cryptic antigens. ${ }^{8}$ Thus, although there are no such indications from immunological data generated after PDT, we still need to investigate whether ROS-based modifications of certain DAMPs by PDT could inactivate them.

It is worth mentioning here that in the context of DAMPs, oxidation might not always be considered to be counter-productive since its effects tend to be biomolecule-dependent and DAMPs (as mentioned previously) are not always protein based in nature. Lipid or lipid-based molecules (e.g. phospholipids) have also been documented to have DAMP-like activity (Table 1). In fact, oxidized phospholipids (Ox-PL) have been documented to be attractive to both humoral and cellular innate immune responses such that their presence on apoptotic cells has been postulated to assist in binding with macrophage scavenger receptors like SR-B1 and CD36 $6^{45,46}$ and in DC maturation regulation. ${ }^{47} \mathrm{Ox}-$ PL have been reported to act as ligands for PRRs and have been found to be capable of activating a TLR4-based signaling pathway independent of NFKB. ${ }^{48}$ In fact, it has been proposed that in the case of phospholipids, oxidation leads to creation of 'new' epitopes recognizable by antibodies. ${ }^{48}$ Observations on Ox-PL and their behavior have led the researchers to postulate the 'Lipid Whisker Model', an extended variant of the Fluid Mosaic Model. ${ }^{49}$ As per the Fluid Mosaic Model, the amphipathic phospholipids are supposed to have their hydrophobic fatty acyl chains hidden within the membrane interior while the hydrophilic polar headgroups are supposed to be oriented towards the aqueous environment. ${ }^{49} \mathrm{How}-$ ever, as per the 'Lipid Whisker Model', when cellular membranes undergo lipid peroxidation (e.g. during apoptosis or inflammation) the resulting oxidation causes previously hydrophobic portions of fatty acids to move towards the aqueous exterior thereby facilitating their recognition by PRRs. ${ }^{49}$ More specifically, it has been observed that the oxidized form of phosphatidylcholine specifically tends to emerge on the apoptotic cells and possibly helps in their clearance by phagocytes. ${ }^{45}$ Researchers have also shown that phosphatidylserine (PS) the 'classical' lipid-based 'eat me' signal (Table 1) tends to bind better to CD36 phagocytosisassisting receptor on macrophages when in oxidized form. ${ }^{50}$ All this taken together means these Ox-PLs present an interesting prospect that deserves further investigation. Interestingly, PDT has been known for some time to cause considerable lipid peroxidation, a process that has been found to play an important role in PDT-mediated cell death and inflammatory processes. ${ }^{51,52}$ Thus, it would be worth investigating if PDT-mediated lipid peroxidation enhances immunogenicity of PDT-treated cells and their phagocytosis.

\section{Cell death accompanied by autophagy and DAMPs: how beneficial can this link be?}

Autophagy accompanying cell death has recently shown the potential to cause 'active' secretion of HMGB1, ${ }^{53}$ a well-known DAMP and the ability to affect the proteome of the cell membrane.,54 Interestingly, it was recently shown that cytosolic HMGB1 is capable of regulating starvation-induced autophagy, thereby further shedding light on a possible link between autophagic processes and HMGB1. ${ }^{55}$ However, it has not been characterized yet if in long run autophagy accompanying cell death helps, in terms of immunological impact. PDT is one of those therapeutic modalities which can convincingly induce cell death accompanied by or caused by autophagy in cancer cells, a subject that has been a topic of many investigations in recent times. ${ }^{31,56-58}$ We envisage that the study of DAMP spectra and immunogenicity in cancer cells that, due to genetic defects in apoptotic pathway, undergo autophagic cell death after PDT could help us in further answering the conundrum of whether autophagy can help by enhancing immunogenicity or reduce it.

\section{DAMPs and their 'alter egos': where does PDT fit in the story?}

The discussions above mostly concentrate on the 'good' facet of DAMPs, i.e. the scenario where DAMPs could help us against diseases like cancer by pro-actively activating the immune system against cancerous lesions. However in recent times it has been revealed that certain DAMPs (especially HMGB1) have an 'alter ego' which might be responsible for them driving certain 
non-infectious inflammatory/autoimmune diseases, e.g. rheumatoid arthritis, systemic lupus erythematosus (SLE), ${ }^{59}$ scleroderma ${ }^{59}$ and renal autoimmune tissue injury. ${ }^{60} \mathrm{~A}$ link has been postulated between the DAMP-associated pro-inflammatory program and the progression of these diseases. However, it is rather hard to fit PDT within this story since PDT that is associated with DAMPs exposure and secretion has actually been in use for treatment of various non-infectious inflammatory/autoimmune diseases with positive results, e.g. psoriasis, ${ }^{61}$ psoriatic arthritis, ${ }^{62}$ rheumatoid arthritis, ${ }^{63,64}$ scleroderma, ${ }^{8,65}$ vulvar lichen sclerosus ${ }^{66}$ and autoimmune skin ulcers. ${ }^{67}$ PDT-based treatment of these diseases has been considered to be promising, meaning that the relationship between DAMPs and inflammatory/autoimmune diseases needs a 'fresh re-look' on the lines of PDT. It is possible that in these settings PDT might be proving to be beneficial due to 'production' of anti-inflammatory DAMPs or by inactivating certain 'problematic' inflammatory DAMPs (possibly via ROS), however both these possibilities need further investigation. Nevertheless, one point is clear with this backdrop: that PDT has considerable beneficial immunomodulatory potential in terms of disease management even if the diseases are originally inflammation-associated/-driven.

\section{Concluding remarks}

DAMP-related research has been around for a considerable time and, while the complete extent and diversity up to which cells can expose DAMPs is still to be characterized, several 'new' DAMPs have emerged recently. Moreover, major effort needs to be invested on getting a better knowledge about the link between a particular DAMP and a particular cell death pathway as well as the molecular pathways underlying the extracellular emergence of DAMPs. Considering the uniqueness of PDT in terms of its settings, and its proven immunogenic character, we can safely assume that PDT holds great promise not only in terms of 'exposing' as yet unknown DAMPs but also in terms of shedding more light on the molecular pathway underlying the translocation/secretion of these DAMPs. Moreover, urgent attention is needed to characterize the presence of immunogenic apoptosis following PDT so that this treatment modality can be optimized for clinical induction of immunogenic cell death in tumours.

\section{Abbreviations}

$\begin{array}{ll}\text { APC } & \text { Antigen-Presenting Cells } \\ \text { ATP } & \text { Adenosine Triphosphate } \\ \text { CDAMP } & \text { Cell Death Associated Molecular Patterns } \\ \text { CRT } & \text { Calreticulin } \\ \text { DAMP } & \text { Damage-Associated Molecular Patterns } \\ \text { DC } & \text { Dendritic Cells } \\ \text { ECM } & \text { Extracellular Matrix } \\ \text { ER } & \text { Endoplasmic Reticulum } \\ \text { HMGB1 } & \text { High Mobility Group Box-1 } \\ \text { HSP } & \text { Heat Shock Proteins } \\ \text { Ox-PL } & \text { Oxidized Phospholipids } \\ \text { PAMP } & \text { Pathogen-Associated Molecular Patterns } \\ \text { PDT } & \text { Photodynamic Therapy } \\ \text { p-ox ER stress } & \text { Photo-oxidative Endoplasmic Reticulum stress } \\ \text { PRR } & \text { Pattern Recognition Receptors }\end{array}$

PS ROS Photosensitiser Reactive Oxygen Species

\section{Acknowledgements}

The work from the author's laboratories was supported by grants from the K.U. Leuven (OT49/06) to P.A. and F.W.O Vlaanderen (G.0728.10). This paper presents research results of the IAP6/18, funded by the Interuniversity Attraction Poles Programme, initiated by the Belgian State, Science Policy Office. D. V. K. is a postdoctoral research fellow of the Fund for Scientific Research Flanders (FWO-Vlaanderen), Belgium. Research in the Vandenabeele group is supported by VIB, Ghent University (GROUP-ID consortium of the UGhent MRP initiative), Research Foundation Flanders (FWO-Vlaanderen) (G.0875.11 and G.0973.11), Federal Research Program IAP 6/18, European Research Program FP6 ApopTrain (MRTN-CT-035624) and FP7 Apo-Sys 200767, and the GROUP-ID consortium of the UGent MRP initiative. P.V. holds a Methusalem grant (BOF09/01M00709) from the Flemish Government. Fig. 1 was produced using Servier Medical Art (www.servier.com) for which the authors would like to acknowledge Servier.

\section{References}

1 M. E. Bianchi, DAMPs, PAMPs and alarmins: all we need to know about danger, J. Leukocyte Biol., 2006, 81, 1-5.

2 A. D. Garg, D. Nowis, J. Golab, P. Vandenabeele, D. V. Krysko and P. Agostinis, Immunogenic cell death, DAMPs and anticancer therapeutics: an emerging amalgamation, Biochim. Biophys. Acta, 2010, 1805, 53-71.

3 S. Y. Seong and P. Matzinger, Hydrophobicity: an ancient damageassociated molecular pattern that initiates innate immune responses, Nat. Rev. Immunol., 2004, 4, 469-478.

4 P. Matzinger, Tolerance, danger, and the extended family, Annu. Rev. Immunol., 1994, 12, 991-1045.

5 J. J. Maher, DAMPs ramp up drug toxicity, J. Clin. Invest., 2009, 119, 246-249.

6 C. S. Calfee and M. A. Matthay, Clinical immunology: Culprits with evolutionary ties, Nature, 2010, 464, 41-42.

7 S. S. Iyer, W. P. Pulskens, J. J. Sadler, L. M. Butter, G. J. Teske, T. K. Ulland, S. C. Eisenbarth, S. Florquin, R. A. Flavell, J. C. Leemans and F. S. Sutterwala, Necrotic cells trigger a sterile inflammatory response through the Nlrp3 inflammasome, Proc. Natl. Acad. Sci. U. S. A., 2009, 106, 20388-20393.

8 A. D. Garg, D. Nowis, J. Golab and P. Agostinis, Photodynamic therapy: illuminating the road from cell death towards anti-tumour immunity, Apoptosis, 2010, 15, 1050-1071.

9 D. V. Krysko and P. Vandenabeele, Clearance of dead cells: mechanisms, immune responses and implication in the development of diseases, Apoptosis, 2010, DOI: 10.1007/s10495-10010-10524-10496.

10 M. Korbelik, PDT-associated host response and its role in the therapy outcome, Lasers Surg. Med., 2006, 38, 500-508.

11 M. Korbelik and J. Sun, Photodynamic therapy-generated vaccine for cancer therapy, Cancer Immunol. Immunother., 2005, 55, 900-909.

12 D. R. Green, T. Ferguson, L. Zitvogel and G. Kroemer, Immunogenic and tolerogenic cell death, Nat. Rev. Immunol., 2009, 9, 353-363.

13 L. Zitvogel, O. Kepp and G. Kroemer, Decoding cell death signals in inflammation and immunity, Cell, 2010, 140, 798-804.

14 L. Zitvogel, O. Kepp, L. Senovilla, L. Menger, N. Chaput and G. Kroemer, Immunogenic tumor cell death for optimal anticancer therapy: the calreticulin exposure pathway, Clin. Cancer Res., 2010, 16, 3100-3104.

15 S. Demaria, E. Pikarsky, M. Karin, L. M. Coussens, Y. C. Chen, E. M. El-Omar, G. Trinchieri, S. M. Dubinett, J. T. Mao, E. Szabo, A. Krieg, G. J. Weiner, B. A. Fox, G. Coukos, E. Wang, R. T. Abraham, M. Carbone and M. T. Lotze, Cancer and inflammation: promise for biologic therapy, J. Immunother., 2010, 33, 335-351. 
16 T. Panaretakis, O. Kepp, U. Brockmeier, A. Tesniere, A. C. Bjorklund, D. C. Chapman, M. Durchschlag, N. Joza, G. Pierron, P. van Endert, J. Yuan, L. Zitvogel, F. Madeo, D. B. Williams and G. Kroemer, Mechanisms of pre-apoptotic calreticulin exposure in immunogenic cell death, EMBO J., 2009, 28, 578-590.

17 T. Verfaillie, A. D. Garg and P. Agostinis, Targeting ER stress induced apoptosis and inflammation in cancer, Cancer Lett., 2010, DOI: 10.1016/j.canlet.2010.07.016.

18 S. Hummasti and G. S. Hotamisligil, Endoplasmic reticulumstress and inflammation in obesity and diabetes, Circ. Res., 2010, 107, 579-591.

19 G. S. Hotamisligil, Endoplasmic reticulum stress and the inflammatory basis of metabolic disease, Cell, 2010, 140, 900-917.

20 A. Kaser and R. S. Blumberg, Endoplasmic reticulum stress and intestinal inflammation, Mucosal Immunol., 2009, 3, 11-16.

21 M. Obeid, A. Tesniere, F. Ghiringhelli, G. M. Fimia, L. Apetoh, J. L. Perfettini, M. Castedo, G. Mignot, T. Panaretakis, N. Casares, D. Metivier, N. Larochette, P. van Endert, F. Ciccosanti, M. Piacentini, L. Zitvogel and G. Kroemer, Calreticulin exposure dictates the immunogenicity of cancer cell death, Nat. Med., 2006, 13, 54-61.

22 R. Q. Peng, Y. B. Chen, Y. Ding, R. Zhang, X. Zhang, X. J. Yu, Z. W. Zhou, Y. X. Zeng and X. S. Zhang, Expression of calreticulin is associated with infiltration of T-cells in stage IIIB colon cancer, World J. Gastroenterol., 2010, 16, 2428-2434.

23 D. Jocham, A. Richter, L. Hoffmann, K. Iwig, D. Fahlenkamp, G. Zakrzewski, E. Schmitt, T. Dannenberg, W. Lehmacher, J. von Wietersheim and C. Doehn, Adjuvant autologous renal tumour cell vaccine and risk of tumour progression in patients with renal-cell carcinoma after radical nephrectomy: phase III, randomised controlled trial, Lancet, 2004, 363, 594-599.

24 M. May, S. Brookman-May, B. Hoschke, C. Gilfrich, F. Kendel, S. Baxmann, S. Wittke, S. T. Kiessig, K. Miller and M. Johannsen, Ten-year survival analysis for renal carcinoma patients treated with an autologous tumour lysate vaccine in an adjuvant setting, Cancer Immunol. Immunother., 2009, 59, 687-695.

25 E. A. Hirschowitz, T. Foody, R. Kryscio, L. Dickson, J. Sturgill and J. Yannelli, Autologous dendritic cell vaccines for non-small-cell lung cancer, J. Clin. Oncol., 2004, 22, 2808-2815.

26 L. M. Liau, R. M. Prins, S. M. Kiertscher, S. K. Odesa, T. J. Kremen, A. J. Giovannone, J. W. Lin, D. J. Chute, P. S. Mischel, T. F. Cloughesy and M. D. Roth, Dendritic cell vaccination in glioblastoma patients induces systemic and intracranial T-cell responses modulated by the local central nervous system tumor microenvironment, Clin. Cancer Res., 2005, 11, 5515-5525.

27 K. Palucka, H. Ueno, G. Zurawski, J. Fay and J. Banchereau, Building on dendritic cell subsets to improve cancer vaccines, Curr. Opin. Immunol., 2010, 22, 258-263.

28 C. L. Chiang, F. Benencia and G. Coukos, Whole tumor antigen vaccines, Semin. Immunol., 2010, 22, 132-143.

29 S. O. Gollnick and C. M. Brackett, Enhancement of anti-tumor immunity by photodynamic therapy, Immunol. Res., 2009, 46, 216-226.

30 A. P. Castano, P. Mroz and M. R. Hamblin, Photodynamic therapy and anti-tumour immunity, Nat. Rev. Cancer, 2006, 6, 535-545.

31 E. Buytaert, M. Dewaele and P. Agostinis, Molecular effectors of multiple cell death pathways initiated by photodynamic therapy, Biochim. Biophys. Acta, 2007, 1776, 86-107.

32 G. Brouckaert, M. Kalai, D. V. Krysko, X. Saelens, D. Vercammen, M. Ndlovu, G. Haegeman, K. D'Herde and P. Vandenabeele, Phagocytosis of necrotic cells by macrophages is phosphatidylserine dependent and does not induce inflammatory cytokine production, Mol. Biol. Cell, 2003, 15, 1089-1100.

33 M. F. Tsan and B. Gao, Heat shock proteins and immune system, J. Leukocyte Biol., 2009, 85, 905-910.

34 A. G. Pockley, Heat shock proteins as regulators of the immune response, Lancet, 2003, 362, 469-476.

35 M. Korbelik, J. Sun and I. Cecic, Photodynamic therapy-induced cell surface expression and release of heat shock proteins: relevance for tumor response, Cancer Res., 2005, 65, 1018-1026.

36 F. Zhou, D. Xing and W. R. Chen, Dynamics and mechanism of HSP70 translocation induced by photodynamic therapy treatment, Cancer Lett., 2008, 264, 135-144.

37 S. Carta, P. Castellani, L. Delfino, S. Tassi, R. Vene and A. Rubartelli, DAMPs and inflammatory processes: the role of redox in the different outcomes, J. Leukocyte Biol., 2009, 86, 549-555.

38 G. Minotti, P. Menna, E. Salvatorelli, G. Cairo and L. Gianni, Anthracyclines: molecular advances and pharmacologic developments in antitumor activity and cardiotoxicity, Pharmacol. Rev., 2004, 56, 185-229.

39 Y. J. Hsieh, C. C. Wu, C. J. Chang and J. S. Yu, Subcellular localization of Photofrin determines the death phenotype of human epidermoid carcinoma A431 cells triggered by photodynamic therapy: when plasma membranes are the main targets, J. Cell. Physiol., 2003, 194, 363-375.

40 A. Szokalska, M. Makowski, D. Nowis, G. M. Wilczynski, M. Kujawa, C. Wojcik, I. Mlynarczuk-Bialy, P. Salwa, J. Bil, S. Janowska, P. Agostinis, T. Verfaillie, M. Bugajski, J. Gietka, T. Issat, E. Glodkowska, P. Mrowka, T. Stoklosa, M. R. Hamblin, P. Mroz, M. Jakobisiak and J. Golab, Proteasome inhibition potentiates antitumor effects of photodynamic therapy in mice through induction of endoplasmic reticulum stress and unfolded protein response, Cancer Res., 2009, 69, $4235-4243$

41 A. D. Garg, T. Verfaillie, N. Rubio, G. B. Ferreira, C. Mathieu and P. Agostinis, Hypericin-PDT treatment of cancer cells leads to surface exposure/extracellular release of DAMPs and activates human immature dendritic cells (abstract), Belg. J. Med. Oncol., 2010, 4, 93-94.

42 A. Rubartelli and M. T. Lotze, Inside, outside, upside down: damageassociated molecular-pattern molecules (DAMPs) and redox, Trends Immunol., 2007, 28, 429-436.

43 C. A. Harrison, M. J. Raftery, J. Walsh, P. Alewood, S. E. Iismaa, S. Thliveris and C. L. Geczy, Oxidation regulates the inflammatory properties of the murine S100 protein S100A8, J. Biol. Chem., 1999, 274, 8561-8569.

44 H. Kazama, J. E. Ricci, J. M. Herndon, G. Hoppe, D. R. Green and T. A. Ferguson, Induction of immunological tolerance by apoptotic cells requires caspase-dependent oxidation of high-mobility group box1 protein, Immunity, 2008, 29, 21-32.

45 M. K. Chang, C. J. Binder, M. Torzewski and J. L. Witztum, C-reactive protein binds to both oxidized LDL and apoptotic cells through recognition of a common ligand: Phosphorylcholine of oxidized phospholipids, Proc. Natl. Acad. Sci. U. S. A., 2002, 99, 13043-13048.

46 E. A. Podrez, E. Poliakov, Z. Shen, R. Zhang, Y. Deng, M. Sun, P. J. Finton, L. Shan, B. Gugiu, P. L. Fox, H. F. Hoff, R. G. Salomon and S. L. Hazen, Identification of a novel family of oxidized phospholipids that serve as ligands for the macrophage scavenger receptor CD36, J. Biol. Chem., 2002, 277, 38503-38516.

47 S. Bluml, S. Kirchberger, V. N. Bochkov, G. Kronke, K. Stuhlmeier, O. Majdic, G. J. Zlabinger, W. Knapp, B. R. Binder, J. Stockl and $\mathrm{N}$. Leitinger, Oxidized phospholipids negatively regulate dendritic cell maturation induced by TLRs and CD40, J. Immunol., 2005, 175, 501508.

48 A. Furnkranz and N. Leitinger, Regulation of inflammatory responses by oxidized phospholipids: structure-function relationships, Curr. Pharm. Des., 2004, 10, 915-921.

49 S. L. Hazen, Oxidized phospholipids as endogenous pattern recognition ligands in innate immunity, J. Biol. Chem., 2008, 283, 15527-15531.

50 M. E. Greenberg, M. Sun, R. Zhang, M. Febbraio, R. Silverstein and S. L. Hazen, Oxidized phosphatidylserine-CD36 interactions play an essential role in macrophage-dependent phagocytosis of apoptotic cells, J. Exp. Med., 2006, 203, 2613-2625.

51 D. Nowis, M. Makowski, T. Stoklosa, M. Legat, T. Issat and J. Golab, Direct tumor damage mechanisms of photodynamic therapy, Acta Biochim. Pol., 2005, 52, 339-352.

52 D. V. Sakharov, E. D. Elstak, B. Chernyak and K. W. Wirtz, Prolonged lipid oxidation after photodynamic treatment. Study with oxidationsensitive probe C11-BODIPY581/591, FEBS Lett., 2005, 579, 12551260.

53 J. Thorburn, H. Horita, J. Redzic, K. Hansen, A. E. Frankel and A. Thorburn, Autophagy regulates selective HMGB1 release in tumor cells that are destined to die, Cell Death Differ, 2008, 16, 175183.

54 L. Baricault, J. A. Fransen, M. Garcia, C. Sapin, P. Codogno, L. A. Ginsel and G. Trugnan, Rapid sequestration of DPP IV/CD26 and other cell surface proteins in an autophagic-like compartment in Caco2 cells treated with forskolin, J. Cell. Sci., 1995, 108(Pt 5), 2109-2121.

55 D. Tang, R. Kang, K. M. Livesey, C. W. Cheh, A. Farkas, P. Loughran, G. Hoppe, M. E. Bianchi, K. J. Tracey, H. J. Zeh, 3rd and M. T. Lotze, Endogenous HMGB1 regulates autophagy, J. Cell Biol., 2010, 190, 881-892.

56 M. Dewaele, W. Martinet, N. Rubio, T. Verfaillie, P. A. de Witte, J. Piette and P. Agostinis, Autophagy pathways activated in response to PDT contribute to cell resistance against ROS damage, J. Cell Mol. Med., 2010, DOI: 10.1111/j.1582-4934.2010.01118.x. 
57 M. Dewaele, H. Maes and P. Agostinis, ROS-mediated mechanisms of autophagy stimulation and their relevance in cancer therapy, Autophagy, 2010, 6, DOI: 10.4161/auto.6.7.12113.

58 J. J. Reiners, Jr., P. Agostinis, K. Berg, N. L. Oleinick and D. Kessel, Assessing autophagy in the context of photodynamic therapy, Autophagy, 2010, 6, 7-18.

59 G. P. Sims, D. C. Rowe, S. T. Rietdijk, R. Herbst and A. J. Coyle, HMGB1 and RAGE in inflammation and cancer, Annu. Rev. Immunol., 2010, 28, 367-388.

$60 \mathrm{H}$. J. Anders and D. O. Schlondorff, Innate immune receptors and autophagy: implications for autoimmune kidney injury, Kidney Int., 2010, 78, 29-37.

61 T. Smits, M. M. Kleinpenning, P. E. van Erp, P. C. van de Kerkhof and M. J. Gerritsen, A placebo-controlled randomized study on the clinical effectiveness, immunohistochemical changes and protoporphyrin IX accumulation in fractionated 5-aminolaevulinic acid-photodynamic therapy in patients with psoriasis, Br. J. Dermatol., 2006, 155, 429436.

62 L. G. Ratkay, J. D. Waterfield and D. W. Hunt, Photodynamic therapy in immune (non-oncological) disorders: focus on benzoporphyrin derivatives, BioDrugs, 2000, 14, 127-135.

63 E. Torikai, Y. Kageyama, E. Kohno, T. Hirano, Y. Koide, S. Terakawa and A. Nagano, Photodynamic therapy using talaporfin sodium for synovial membrane from rheumatoid arthritis patients and collageninduced arthritis rats, Clin. Rheumatol., 2007, 27, 751-761.

64 A. Hansch, O. Frey, M. Gajda, G. Susanna, J. Boettcher, R. Brauer and W. A. Kaiser, Photodynamic treatment as a novel approach in the therapy of arthritic joints, Lasers Surg. Med., 2008, 40, 265-272.

65 H. Takahashi, S. Komatsu, M. Ibe, A. Ishida-Yamamoto, S. Nakajima, I. Sakata and H. Iizuka, ATX-S10(Na)-PDT shows more potent effect on collagen metabolism of human normal and scleroderma dermal fibroblasts than ALA-PDT, Arch. Dermatol. Res., 2006, 298, 257-263.

66 A. Olejek, K. Steplewska, A. Gabriel, I. Kozak-Darmas, A. Jarek, S. Kellas-Sleczka, F. Bydlinski, K. Sieron-Stoltny, S. Horak, A. Chelmicki and A. Sieron, Efficacy of Photodynamic Therapy in Vulvar Lichen Sclerosus Treatment Based on Immunohistochemical Analysis of CD34, CD44, Myelin Basic Protein, and Ki67 Antibodies, Int. J. Gynecol. Cancer, 2010, 20, 879-887.

$67 \mathrm{~S}$. Motta and M. Monti, Photodynamic therapy-a promising treatment option for autoimmune skin ulcers: a case report, Photochem. Photobiol. Sci., 2007, 6, 1150-1151.

68 M. Subbarayan, U. O. Hafeli, D. K. Feyes, J. Unnithan, S. N. Emancipator and H. Mukhtar, A simplified method for preparation of $99 \mathrm{mTc}$-annexin $\mathrm{V}$ and its biologic evaluation for in vivo imaging of apoptosis after photodynamic therapy, J. Nucl. Med., 2003, 44, 650656.

69 S. Suzuki and A. B. Kulkarni, Extracellular heat shock protein HSP90beta secreted by MG63 osteosarcoma cells inhibits activation of latent TGF-beta1, Biochem. Biophys. Res. Commun., 2010, 398, 525531.

70 J. R. Riddell, X. Y. Wang, H. Minderman and S. O. Gollnick, Peroxiredoxin 1 stimulates secretion of proinflammatory cytokines by binding to TLR4, J. Immunol., 2009, 184, 1022-1030.

71 A. Iwata, V. Morgan-Stevenson, B. Schwartz, L. Liu, J. Tupper, X. Zhu, J. Harlan and R. Winn, Extracellular BCL2 proteins are dangerassociated molecular patterns that reduce tissue damage in murine models of ischemia-reperfusion injury, PLoS One, 2010, 5, e9103.

72 K. E. Blume, S. Soeroes, M. Waibel, H. Keppeler, S. Wesselborg, M. Herrmann, K. Schulze-Osthoff and K. Lauber, Cell surface externalization of annexin $\mathrm{A} 1$ as a failsafe mechanism preventing inflammatory responses during secondary necrosis, J. Immunol., 2009, 183, 8138-8147.

73 A. A. Manfredi and P. Rovere-Querini, The mitochondrion-a Trojan horse that kicks off inflammation?, N. Engl. J. Med., 2010, 362, 21322134.

74 Q. Zhang, M. Raoof, Y. Chen, Y. Sumi, T. Sursal, W. Junger, K. Brohi, K. Itagaki and C. J. Hauser, Circulating mitochondrial DAMPs cause inflammatory responses to injury, Nature, 2010, 464, 104-107.

75 A. Babelova, K. Moreth, W. Tsalastra-Greul, J. Zeng-Brouwers, O. Eickelberg, M. F. Young, P. Bruckner, J. Pfeilschifter, R. M. Schaefer, H. J. Grone and L. Schaefer, Biglycan, a danger signal that activates the NLRP3 inflammasome via toll-like and P2X receptors, J. Biol. Chem., 2009, 284, 24035-24048.

76 L. Schaefer, Extracellular matrix molecules: endogenous danger signals as new drug targets in kidney diseases, Curr. Opin. Pharmacol., 2010, 10, 185-190.

77 F. G. Goh, A. M. Piccinini, T. Krausgruber, I. A. Udalova and K. S. Midwood, Transcriptional regulation of the endogenous danger signal tenascin-C: a novel autocrine loop in inflammation, J. Immunol., 2010, 184, 2655-2662.

78 T. E. Ichim, Z. Zhong, S. Kaushal, X. Zheng, X. Ren, X. Hao, J. A. Joyce, H. H. Hanley, N. H. Riordan, J. Koropatnick, V. Bogin, B. R. Minev, W. P. Min and R. H. Tullis, Exosomes as a tumor immune escape mechanism: possible therapeutic implications, J. Transl. Med., $2008,6,37$.

79 L. Zitvogel, A. Tesniere and G. Kroemer, Cancer despite immunosurveillance: immunoselection and immunosubversion, Nat. Rev. Immunol., 2006, 6, 715-727. 